\title{
Modeling Cardiac Electromechanics and Mechanoelectrical Coupling in Dyssynchronous and Failing Hearts
}

\author{
Insight from Adaptive Computer Models
}

\author{
Nico H. L. Kuijpers • Evelien Hermeling • \\ Peter H. M. Bovendeerd • Tammo Delhaas • \\ Frits W. Prinzen
}

Received: 13 November 2011 / Accepted: 4 January 2012 / Published online: 21 January 2012

(C) The Author(s) 2012. This article is published with open access at Springerlink.com

\begin{abstract}
Computer models have become more and more a research tool to obtain mechanistic insight in the effects of dyssynchrony and heart failure. Increasing computational power in combination with increasing amounts of experimental and clinical data enables the development of mathematical models that describe electrical and mechanical behavior of the heart. By combining models based on data at the molecular and cellular level with models that describe organ function, so-called multi-scale models are created that describe heart function at different length and time scales. In this review, we describe basic modules that can be identified in multi-scale models of cardiac electromechan-
\end{abstract}

N. H. L. Kuijpers $(\bowtie) \cdot$ E. Hermeling · T. Delhaas

Department of Biomedical Engineering,

Maastricht University, Maastricht, The Netherlands

e-mail: nico.kuijpers@maastrichtuniversity.nl

E. Hermeling

e-mail: e.hermeling@maastrichtuniversity.nl

T. Delhaas

e-mail: tammo.delhaas@maastrichtuniversity.nl

P. H. M Bovendeerd

Department of Biomedical Engineering,

Eindhoven University of Technology, Eindhoven,

The Netherlands

e-mail: P.H.M.Bovendeerd@tue.nl

\section{F. W. Prinzen}

Department of Physiology, Maastricht University,

Maastricht, The Netherlands

e-mail: frits.prinzen@maastrichtuniversity.nl ics. These modules simulate ionic membrane currents, calcium handling, excitation-contraction coupling, action potential propagation, and cardiac mechanics and hemodynamics. In addition, we discuss adaptive modeling approaches that aim to address long-term effects of diseases and therapy on growth, changes in fiber orientation, ionic membrane currents, and calcium handling. Finally, we discuss the first developments in patientspecific modeling. While current models still have shortcomings, well-chosen applications show promising results on some ultimate goals: understanding mechanisms of dyssynchronous heart failure and tuning pacing strategy to a particular patient, even before starting the therapy.

Keywords Adaptive modeling $\cdot$ Cardiac dyssynchrony $\cdot$ Heart failure $\cdot$ Mechanoelectrical coupling $\cdot$ Multi-scale model $\cdot$ Patient-specific model

\section{Introduction}

During the last decade, "dyssynchronous heart failure" has become recognized as a special form of heart failure. Patients with heart failure and "dyssynchrony" respond differently to various therapies, including cardiac resynchronization therapy (CRT) [72, 92]. Dyssynchrony has become the term that is used for either non-synchronous, abnormal electrical activation, and/or an abnormal sequence of contraction. Better understanding of this disease and its therapy has been achieved using animal experiments [34, 71]. However, such experiments have the drawback that within the same animal, only a limited number of parameters can 
be easily manipulated and/or measured and that the biological variation (in time and between animals) can obscure effects. With increasing knowledge of heart failure in general and dyssynchronous heart failure in particular, it becomes harder to grasp the interrelation of the physiological and pathological processes that are involved in the progression of the disease.

Here, mathematical models may become useful. Mathematical models are based on experimentally determined material properties and known physical laws. They are an increasingly powerful instrument to understand cardiac physiology and to integrate knowledge. After all, computer power is growing, efficient algorithms are being developed, and the amount of data on molecular and cellular mechanisms is exponentially growing. The first relevant mathematical models described skeletal muscle mechanics and date from the 1930s [20]. Present models of cardiac mechanics are still based on those equations. A similar observation can be made for electrophysiology [66]. The 1952 Hodgkin and Huxley model of the action potential of the squid giant axon [21] has formed the basis for later models describing cardiac electrophysiology. Subsequently, models based on data at the molecular and cellular level were combined with models that describe organ function to form a multi-scale model, an approach that more recently became known as "systems biology." Such models are based on biological knowledge across physical scales in size (from molecule to organ) and time (milliseconds to months). Physicochemical principles are applied together with engineering methods to develop structurally and functionally integrative predictive models of the dynamic physiology of living systems in health and disease. In this way, models may help to integrate knowledge from a large number of measurements, to test new hypotheses about mechanisms of disease, and to investigate potential clinical interventions. Moreover, models can be made patientspecific by feeding a model with data derived from a particular patient.

We will discuss the various models and focus at a novel approach: to also include (patho)physiological adaptations in the model. After all, the human body is not static, but may change structure and function over time, due to a disease process or a therapy. In particular, we will address the complicated coupling between electrical activation and mechanics and the adaptation in the reverse direction: mechanoelectrical feedback (MEF). Finally, we will discuss the impact of adaptive modeling for computer models that are aimed at describing individual patients, i.e., patient-specific modeling.

\section{Modeling of Cardiac Electromechanics}

Modeling dyssynchronous heart failure is a challenging problem. In order to simulate this condition accurately, reliable data on anatomy of muscle structure as well as the conduction system, ionic membrane currents, calcium handling, and excitation-contraction coupling (ECC) is required. To assess pump function of the heart, cardiac preload and afterload should be incorporated through a model of the circulation. Traditionally, large-scale geometrical computer models of the heart were either aimed at cardiac electrophysiology [30, $67,83,87,96]$ or at cardiac mechanics [10, 52]. Most models describing both cardiac electrophysiology and mechanics are weakly coupled, i.e., electrophysiology (in particular activation sequences) is computed separately from mechanical behavior [17, 31, 32, 90, 91]. Some models are strongly coupled, i.e., at cellular level, a direct link between electrophysiology and mechanics is established by incorporating ECC [8]. These models are multi-scale in nature; mechanics and electrophysiology are modeled at cellular level and are incorporated in a geometrical model of the heart to investigate behavior at organ level $[53,85]$. By incorporating stretch-activated channels [37, 41], the effects of mechanoelectrical feedback have been investigated [26, 29, 44]. In addition to electrophysiology and mechanics, some models also incorporate fluid dynamics of the blood [59].

The research question to be addressed determines the choice of the level of detail each of these aspects has to be incorporated. We summarize published models and their relevance when modeling dyssynchrony or heart failure.

Ionic Membrane Currents and Calcium Handling In 1952, Hodgkin and Huxley published their model of the action potential of the squid giant axon [21]. Still, ionic membrane currents in cardiac membrane models are based on the principles introduced by Hodgkin and Huxley [66]. Mechanical contraction is mediated by an increase in intracellular calcium concentration. Most of the calcium used during contraction is stored in the sarcoplasmic reticulum (SR) in the myocyte. Upon electrical activation, calcium is released from the SR by calcium entering through the cell membrane. This process is called calcium-induced calcium release [8] and thus involves the interaction between ionic membrane currents and SR currents. Because of this strong interrelationship, calcium handling is considered part of modern cardiac membrane models [16, 50]. Human ventricular action potentials and calcium handling are 
described by the models of Iyer et al. [24], Ten Tusscher et al. [88, 89], Grandi et al. [15], and O'Hara et al. [61]. Human atrial action potentials are described by the models of Nygren et al. [60], Courtemanche et al. [13], and Grandi et al. [14].

The calcium transient in failing myocytes is characterized by a smaller amplitude and a longer duration, as explained by reduced uptake of calcium by the SR. The effect is partly compensated by an increased activity of the sodium-calcium exchanger current [63, 64]. Also the action potential is prolonged due to a reduction of repolarizing potassium currents. By incorporating these changes in cardiac membrane models, models of failing myocytes have been developed [68, 97].

Excitation-Contraction Coupling Cross bridges bind upon calcium release. The myocyte contracts by consecutive attachment and detachment of these cross bridges. The first model describing cross-bridge cycling was published in 1957 by Huxley [23]. Later models are based on similar principles but differ in the description of actin-myosin interactions [11, 74, 85]. Models of $\mathrm{Ca}^{2+}$-force relation that have been applied in multiscale models of the human heart include those by Rice et al. [75, 76] and Niederer et al. [54, 58]. Contractile force computed from these models is related to the intracellular concentration of free calcium and sarcomere length. By modeling calcium handling as observed in failing myocytes (e.g., $[68,97])$, multi-scale heart failure models can be created.

Action Potential Propagation In whole-heart electromechanical models, propagation of the action potential throughout the cardiac muscle must be incorporated, because regional differences in electrical activation have a large influence on mechanical behavior. Electrophysiological properties of cardiac tissue are most accurately described when a distinction is made between the intracellular and interstitial domains. In the socalled bidomain equations $[18,86]$, conductive properties of cardiac tissue are modeled as a continuous twophase medium. Under the assumption that the ratio of anisotropy of the intracellular and interstitial domains is equal, the bidomain equations can be reduced to a monodomain description [28]. This approach leads to a considerable reduction in computational effort when modeling action potential propagation through the human ventricles [67]. If only impulse propagation is to be computed, the eikonal diffusion equation can be used. This equation is derived from the bidomain model and solves only for activation times [12]. This approach is computationally less demanding and has been successfully applied in finite-element models of the heart that focus on mechanics [31, 32].

Cardiac Mechanics The deformation of the heart during the cardiac cycle is determined by the mechanical equilibrium between forces related to active contraction of the myofibers, passive stretch of the connective tissue matrix, pressure in the cardiac cavities, and pressure exerted by the pericardium. The finite-element method is often employed to solve the equilibrium equations. Current finite-element models account for the thick-walled nature of the cardiac walls and the varying orientation of the myofibers across the cardiac walls [33]. The mechanical stresses in the tissue are modeled as the summation of a passive and an active stress component. Models for the passive stress component mostly assume the myocardium to behave as incompressible material. The microstructure of the myocardium, in which myocytes are organized in "sheets" of about four cells thick [45], is reflected in the assumption of orthotropic behavior; properties along the fiber direction, perpendicular to the fiber but within the sheet, and perpendicular to the sheet are modeled differently.

It is the active stress component through which models of cardiac mechanics and cardiac electrical activity are coupled. The temporal change of the active stress can be described by the models for calcium release and excitation-contraction coupling described above. The spatial distribution of the moment of onset of calcium release is described by action potential propagation models. A fully coupled solution of the equations involved is highly computationally demanding. Therefore, often weakly coupled approaches are and have been used [17, 31, 32]. For example, the sequence of electrical activation has been determined using an eikonal diffusion model $[31,32]$ that was derived from the bidomain model [12]. In a more advanced approach, the two-variable modified FitzHugh-Nagumo equations that describe membrane behavior were coupled with a monodomain model for impulse propagation [91]. The spatial maps of activation times thus created were subsequently used to initiate active stress development. Active stress was modeled phenomenologically to depend on sarcomere length, sarcomere shortening velocity, and time elapsed since the moment of activation. Gradually, strongly coupled approaches appear that employ a mono-domain formulation for electrical activity $[1,57]$.

When applied to the case of asynchronous activation, models of cardiac electromechanics were able to simulate the characteristic relation between activation 
and mechanics that has also been observed in experiments $[69,70]$. In early-activated regions, a rapid early systolic fiber shortening was found, followed by strongly reduced late systolic shortening. Lateractivated regions were characterized by early systolic lengthening followed by pronounced systolic shortening $[31,91]$.

Several model studies of cardiac mechanics have indicated that myofiber orientation is an important determinant of local myocardial tissue stress and strain [7,9]. In particular, it was demonstrated that, with a suitable choice of myofiber orientation within the range of anatomical findings, fiber stress and strain during ejection are likely to be distributed homogeneously across the wall. Based on this finding, the so-called one-fiber model was developed, in which fiber stress and strain are approximated by single values that are assumed to be valid for the whole wall [4]. On the basis of this approach, the CircAdapt model relates myofiber stress and myofiber stretch ratio to left ventricular pressure and cavity volume [5]. In the current version of the CircAdapt model [49], a separate description of mechanics and adaptation of the septum and the free walls of the left and right ventricle is employed to describe the interaction between the left and right ventricle. In principle, the reduction of spatial detail in these models allows for a more extensive coupling of cardiac electromechanics from the subcellular level to the organ level.

\section{Modeling Adaptation of Cardiac Structure and Mechanics}

It is well-known from clinical studies as well as animal experiments that longer-lasting abnormalities in mechanical loading of the heart lead to structural and functional changes. One example is the increase in muscle mass (hypertrophy) in response to pressure overload, which is often accompanied by molecular and cellular changes. Current models aim at describing either the healthy heart or a certain state of disease, but for a real understanding of the processes underlying progressive diseases such as heart failure, the transition from one state to another is of importance. This has already been recognized in experimental research in which the effect on heart function of a single intervention, e.g., the artificial creation of left bundle branch block (LBBB), is assessed over a longer period of time [93, 94]. In these and other experiments, it was observed that dyssynchronous contraction of the left ventricle eventually leads to changes in wall volume [93] and, at the cellular level, to changes in ionic membrane currents [27] and calcium handling [2]. Processes underlying these changes in (local) behavior are termed structural, electrical, or mechanical remodeling. In the case of chronic derangements, such as dyssynchrony or hypertension, remodeling may not be able to offer complete compensation but will lead instead to heart failure.

At present, the underlying remodeling processes that lead to heart failure are not well understood, because the various processes are strongly interrelated. Mathematical models that include adaptation may provide more insight in the interaction of remodeling processes and their effects on heart function, since they enable selective blocking of these interactions. Thus, we arrive at a new generation of cardiac models in which the time evolution is not only described during a single heart beat but also at the time scale of many weeks, required for the remodeling processes to conclude. In the latter, so-called adaptive modeling approach, model parameters are adjusted each cardiac cycle until steady-state is reached. Running these models typically requires 50 to 150 cardiac cycles, thus demanding much more computational power compared to traditional models that reach steady state within 5 to 10 cycles.

Dyssynchronous heart failure is often accompanied by LBBB that leads to a strongly delayed activation of the left ventricular free wall. Therefore, the minimal requirement of a model to simulate dyssynchrony is to distinguish between left ventricular free wall, septum, and right ventricular free wall. The CircAdapt model, shown in Fig. 1, is designed this way. It comprises a number of basic modules that can be configured to form a network [5, 49]. Each of these modules belongs to one of the following main categories: (1) tube, (2) wall segment, (3) valve, and (4) resistance. Together, these modules represent the heart and the systemic and pulmonary circulations. The model incorporates both the direct mechanical interaction of the left and right ventricle through the interventricular septum and the indirect hemodynamic interaction between both ventricles through the systemic and pulmonary circulation. Global ventricular pump mechanics (pressure-volume relation) are related to myofiber mechanics (myofiber stress-strain relation) on the basis of the principle of conservation of energy. Active force generated by the myofibers is related to time of excitation, sarcomere length, and sarcomere shortening velocity using an empirical model [49].

An important aspect of the CircAdapt model is that material parameters can be automatically adapted to obtain physiological behavior of heart and vessel walls. For example, cardiac wall volume is adapted to obtain an optimal sarcomere length at end diastole. In a similar fashion, cavity volume is adapted on the basis of sarcomere shortening during ejection. Also, vascular 


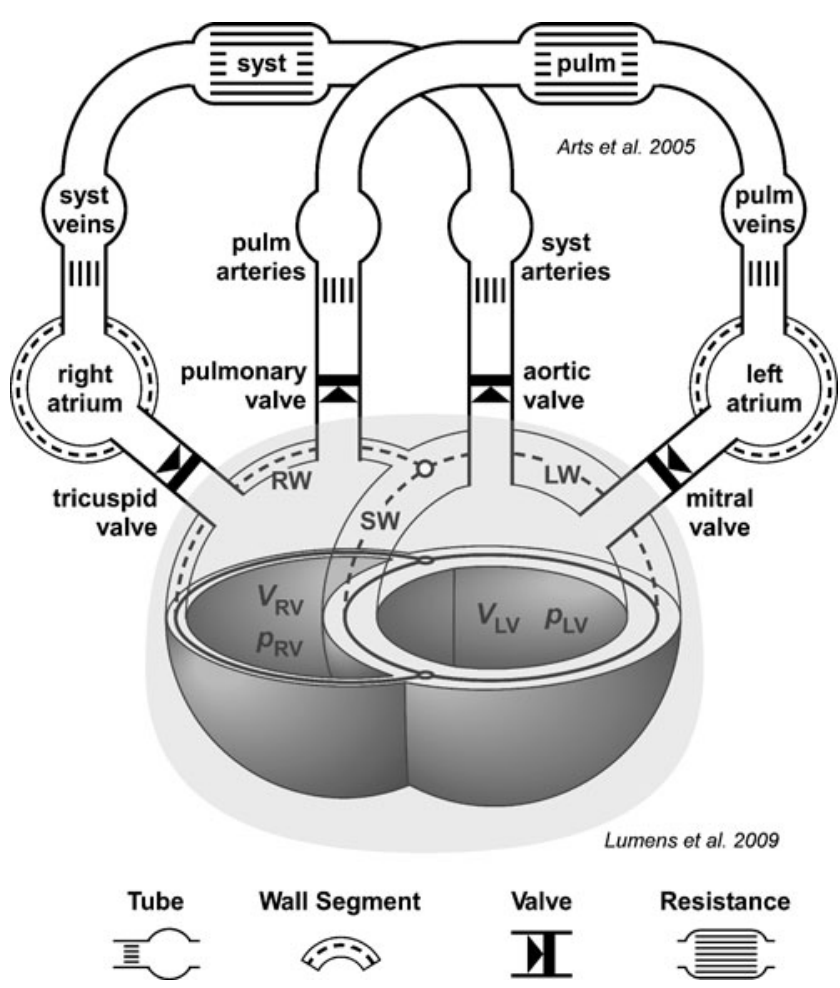

Fig. 1 Schematic overview of the CircAdapt model. Cardiac hemodynamics was modeled by placing the ventricles in a systemic and pulmonary circulation including atria, valves, arteries, organs, and veins. Properties of the right ventricular wall (RW), septal wall (SW), and left ventricular wall (LW) can be modified independently in order to simulate left bundle branch block. Adapted from Lumens et al. [49]

material properties are adapted to shear rate and wall stress (see Lumens et al. [49] for constraints on these parameters). With this approach, only a few adaptation rules are required to obtain a physiological circulation or to simulate the transition from the fetal heart and circulation to that of a newborn [5]. The model also allows to simulate pathology. For instance, acute pulmonary arterial hypertension (PAH) was simulated by increasing the pulmonary resistance. A simulation of compensated PAH was achieved by automatic adaptation of model parameters to the increased resistance [47].

For a spatially more detailed description of myocardial growth, continuum mechanics formulations are needed. Many three-dimensional growth models are based on the models proposed by Skalak et al. [79] and Rodriguez et al. [77]. In these models, a growth stimulus is translated into a growth tensor that describes the growth of infinitesimal tissue elements in a virtual, isolated state. To join the grown elements into a continuous structure, an additional elastic deformation is needed. This elastic deformation is believed to cause the residual stress observed in the heart and other biological tissues [62]. In a series of studies, the group of Taber employed this framework to describe the embryonic growth in geometrically simple models of the chick ventricle $[46,81,82]$. In particular, the longterm stability of several growth laws was investigated. Rausch et al. [73] adopted the approach proposed by Skalak et al. [79] and Rodriguez et al. [77] to model growth in response to hypertension in a biventricular model with a realistic fiber architecture. Here, longterm stability problems were avoided by limiting the maximum tissue growth allowed, which is in agreement with the physiological limitation of cell growth.

The aforementioned growth studies consider the tissue as a one-component material with a fixed stressfree reference state. As an alternative, Humphrey and Rajagopal [22] proposed the constrained mixture approach. In this approach, tissue components are assumed to be in a state of continuous turnover, and new components can be created at a stress-free state that differs from that of the old components. In this view, residual stress results from the difference in the evolving stress-free state of the components. Kroon et al. [39] investigated the consequences of using either a fixed reference state or an evolving reference state in simulating cardiac growth and found that the stimulus for growth decreased over time for the updated reference state, but increased for the fixed reference state. Thus, it was concluded that the choice of the reference configuration affects the stability of the growth process.

As mentioned before, in models of cardiac mechanics, it was demonstrated that myofiber orientation is an important determinant of the spatial distribution of mechanical load across the cardiac walls. This finding suggests that reorientation is an attractive mechanism for the myocyte to optimize the mechanical load that it experiences. The first model of adaptive remodeling of fiber orientation was proposed by Arts et al. [6]. In this model, the left ventricle was modeled through a set of nested cylindrical shells, each with a specific fiber orientation. It was assumed that, in each shell, fiber orientation changed continuously, until sarcomere length at begin ejection and sarcomere shortening during ejection reached preset optimum values. The resulting transmural distribution of fiber orientation varied continuously around a stationary average and was in agreement with anatomical findings.

Kroon et al. [40] developed a model for remodeling of fiber orientation in a general three-dimensional cardiac geometry. It was assumed that, during the cardiac cycle, the structural integrity of the myocardium is continuously challenged by fiber cross-fiber shear deformation. At the same time, the process of 
continuous turnover of the extracellular matrix (ECM) was assumed to lead to rearrangement of connections in between myocytes, such that forces between the myofibers and the ECM would be reduced. Eventually, a structure would appear with minimal fiber cross-fiber shear. This conceptual model was translated into a mathematical formulation, in which the fiber orientation gradually evolved toward one of the eigenvectors of the right Cauchy-Green strain tensor. Upon adaptation, a realistic fiber field was obtained. Local measures of tissue function, like maximum fiber stress, fiber shortening during ejection, and stroke work density, increased by at least $10 \%$ and became more homogeneously distributed across the wall. Measures of pump function, like peak systolic pressure, stroke volume, and stroke work, increased significantly as well.

\section{Modeling Adaptation of Cardiac Electrophysiology}

The adaptive response of the heart is not limited to changes in size and tissue structure, but may also involve changes in electrical behavior. Such adaptations can be observed as T-wave changes in the surface electrocardiogram when returning to normal sinus rhythm after a period of ventricular pacing [78]. This phenom- enon is known as "T-wave memory" and has been linked to changes in membrane currents, including the L-type calcium current [2, 65]. Evidence is growing that mechanical stimuli are involved in the mechanisms behind T-wave memory $[25,38,80]$. As mentioned above, asynchronous activation leads to an inhomogeneous spatial distribution of fiber strain, fiber stress, and regional mechanical work [70].

On the basis of these experimental observations, Kuijpers et al. [42, 43] developed a model to investigate whether deviation from normal work load could function as a trigger for electrical remodeling in the left ventricle. In Fig. 2, their modeling approach is shown, which was used to investigate effects of MEF on cardiac function and dispersion of repolarization both in the asynchronous heart and after resynchronization. Electromechanical behavior of the left ventricular wall was described by a single cardiac fiber that was composed of 300 segments that were both electrically and mechanically coupled [19, 41, 42]. Each of the segments comprised ionic membrane currents, calcium handling, and ECC. Heterogeneity within the fiber was accomplished by gradually varying potassium currents $I_{\mathrm{to}}$ and $I_{\mathrm{Ks}}$, such that action potential durations were the longest in the early-activated regions and gradually decreased toward the later-activated regions. Fiber stress and strain were converted to left ventricular

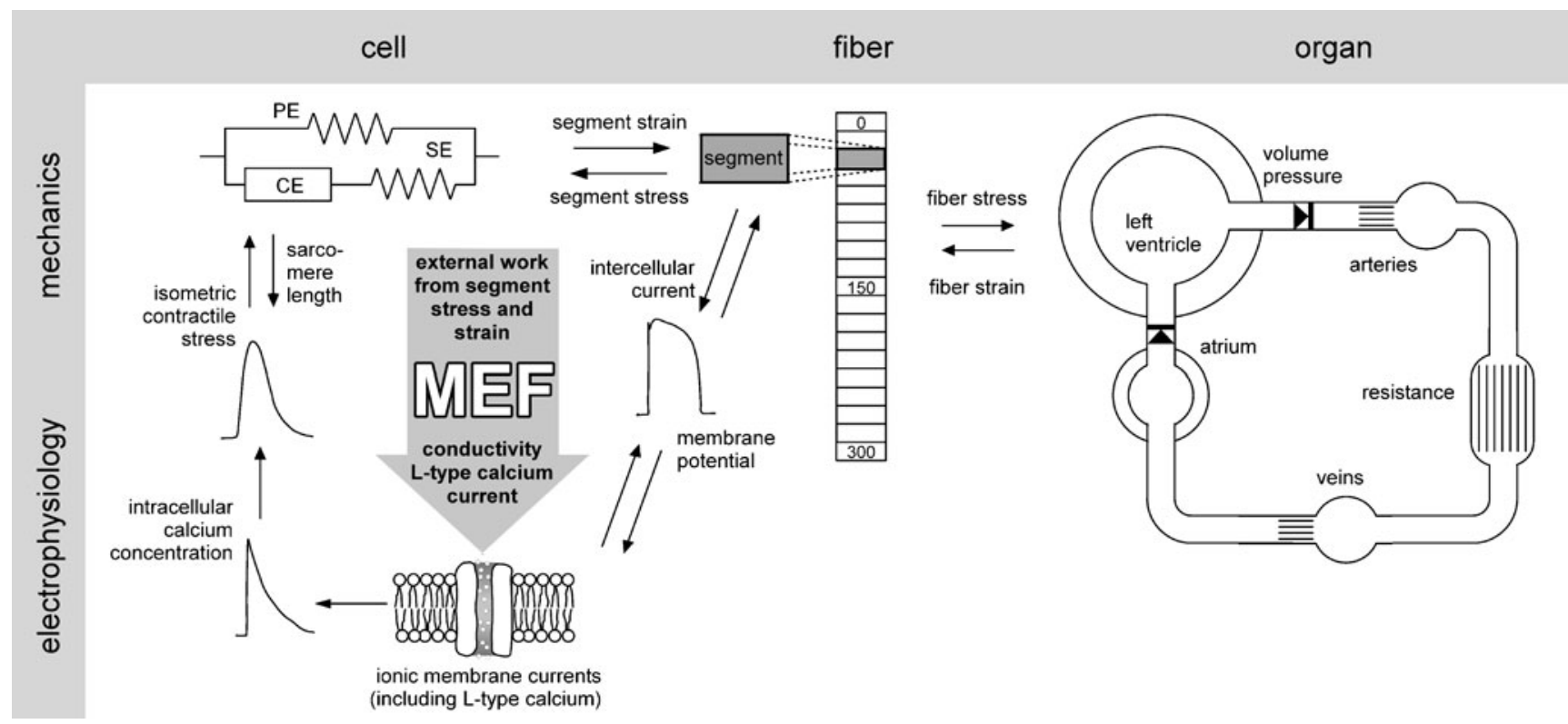

Fig. 2 Schematic overview of the model of electrical remodeling proposed by Kuijpers et al. [42, 43]. Cardiac electromechanics was described by a single fiber composed of 300 segments. Left ventricular pressure and volume were related to fiber stress and strain. Cardiac hemodynamics was modeled by placing the left ventricle in a systemic circulation describing the left atrium, valves, arteries, organs, and veins. Electrical activation was started by activating one (normal, LBBB) or both (CRT) fiber ends. Mechanoelectrical feedback (MEF) was incorporated by adjusting segmental L-type calcium current $\left(I_{\mathrm{CaL}}\right)$ to obtain target myofiber external work 
blood pressure and volume, respectively, according to the one-fiber model [3]. To obtain physiological pressure-volume relations, systemic blood circulation was modeled by the CircAdapt model [5]. MEF was incorporated by adjusting segmental L-type calcium current to obtain target myofiber external work. With this model, the effect of MEF in LBBB as well as with CRT was evaluated. In Fig. 3, membrane potential $\left(V_{\text {mem }}\right)$, intracellular calcium concentration $\left(\left[\mathrm{Ca}^{2+}\right]_{\mathrm{i}}\right)$, and strain excursions are shown for three segments in five subsequent simulation runs.

In the normal heart (activation time $30 \mathrm{~ms}$ ), the opposite directions of depolarization and repolarization are caused by heterogeneity in potassium currents $I_{\mathrm{to}}$ and $I_{\mathrm{Ks}}$ and lead to T-wave concordance as well as simultaneous relaxation of the three segments. With acute LBBB (activation time $108 \mathrm{~ms}$ ), repolarization occurs simultaneously for the three segments, but strain excursions are more asynchronous than in the normal heart. With chronic LBBB, MEF leads to less asynchronous strain excursions due to increased calcium influx in early-activated segments. However, upon acute CRT (activation from both sides of the fiber, activation time $54 \mathrm{~ms}$ ), contractile behavior of the two early- activated segments differ largely due to MEF-induced remodeling during chronic LBBB. In chronic CRT, this difference is compensated for by the ongoing MEF. In Fig. 4, dispersion in external work $\left(W_{\text {ext }}\right)$, action potential duration $\left(\mathrm{APD}_{-60 \mathrm{mV}}\right)$, and repolarization $\left(t_{\text {repol }}\right)$ are shown during acute and chronic LBBB and during acute and chronic CRT. Due to MEF, dispersion in $W_{\text {ext }}$ was decreased, both during chronic LBBB and CRT. Note, however, that dispersion of repolarization acutely increases with onset of CRT but reduces during chronic CRT due to MEF. In the acute phase, CRT may thus lead to an increased dispersion of repolarization, leading to impaired filling and possibly increased risk of ventricular arrhythmia [51]. Indeed, in a patient study, such repolarization changes have been observed, reflected as development of cardiac memory within 2 weeks of CRT [95]. With time, repolarization becomes more synchronized, and diastolic heart function improves due to mechanoelectrical feedback. Adaptation of cardiac electrophysiology may also be incorporated in three-dimensional models of cardiac electrophysiology to predict long-term effects of dyssynchrony and pacing strategies in different regions of the heart.
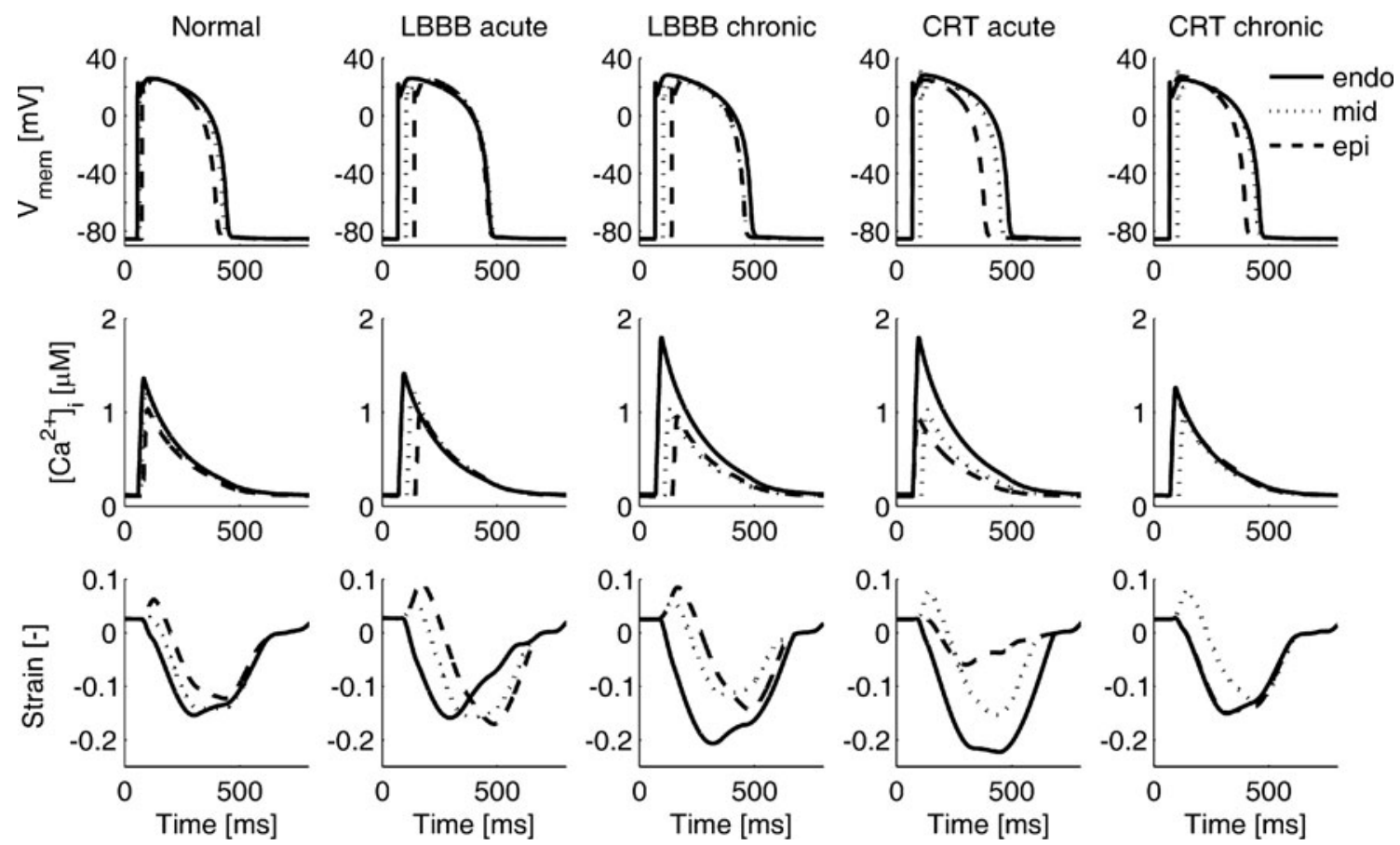

Fig. 3 Membrane potential $\left(V_{\text {mem }}\right)$, calcium transient $\left(\left[\mathrm{Ca}^{2+}\right]_{\mathrm{i}}\right)$, and strain for three segments located at the endocardium, midwall, and epicardium of the ventricular wall. Results are shown for all five subsequent simulations, representing the transition

from the healthy to the chronically diseased state in left bundle branch block (LBBB) and its response to cardiac resynchronization therapy (CRT) on the short and long term. Modified from Kuijpers et al. [43] 
Fig. 4 Dispersion in external work $\left(W_{\text {ext }}\right)$, action potential duration (APD_60mv), and repolarization $\left(t_{\text {repol }}\right)$ during left bundle branch block (LBBB) and cardiac resynchronization therapy (CRT). Modified from Kuijpers et al. [43]
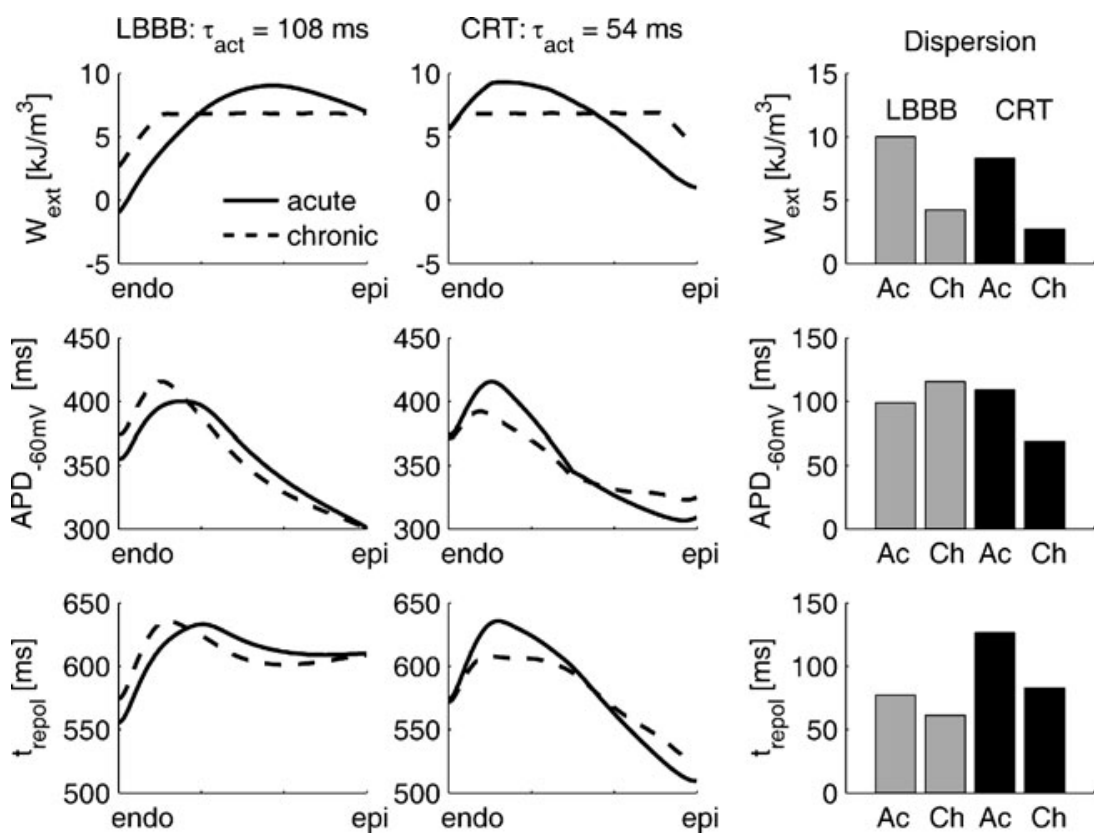

\section{Clinical Applications}

At present, models of electromechanical behavior of dyssynchronous hearts are multi-scale by nature and describe electrophysiology, excitation-contraction coupling, mechanical function, and hemodynamics [34$36,85]$. Various groups have made the first steps in converting generic models into patient-specific models, in order to create a diagnostic and decision support tool for the cardiologist $[1,55,56,84]$. Often, a detailed geometrical representation is aimed at, and consequently, the finite-element method is used to solve the mechanical equilibrium equations. Geometrical input data for such models may be extracted directly from clinical measurements. However, other input data, e.g., on the spatial distribution of tissue electromechanical properties, cannot be determined from direct clinical measurements. Instead, they must be determined in an iterative procedure, in which model response is matched to observed clinical response. Both this optimization procedure and the finite-element method are time-consuming. However, clinical application seems to come within reach: in a recent strongly coupled patientspecific model, the computation of cardiac electromechanics during one cardiac cycle only required about 3 to $4 \mathrm{~h}$ on a desktop PC, thanks to the use of GPU programming.

In that regard, the CircAdapt model $[5,49]$ may be a useful alternative. Indeed, in this model, the first steps toward patient-specific modeling in PAH have led to a better understanding of left ventricular inflow patterns in $\mathrm{PAH}$ as well as to the interesting observation that right ventricular pacing in PAH might be beneficial [47, 48]. Computation times are limited thanks to the geometric simplicity of the model. But, more importantly, many electromechanical tissue properties are derived from applying knowledge on physiological and pathophysiological adaptation mechanisms, thus eliminating the need for a time-consuming optimization procedure. As shown in this review, the phenomenological approach to model excitation-contraction coupling in CircAdapt may be replaced with a cascade of physiological models to investigate the effects of electrical remodeling on cardiac pump function. By extending geometrically more realistic patient-specific models of dyssynchronous heart failure with structural and electrical adaptation, a more accurate prediction of longterm effects of pacing therapy for a particular patient will become feasible clinically.

Open Access This article is distributed under the terms of the Creative Commons Attribution Noncommercial License which permits any noncommercial use, distribution, and reproduction in any medium, provided the original author(s) and source are credited.

\section{References}

1. Aguado-Sierra, J., Krishnamurthy, A., Villongco, C., Chuang, J., Howard, E., Gonzales, M. J., et al. (2011). Patient-specific modeling of dyssynchronous heart failure: A case study. Progress in Biophysics and Molecular Biology, 107, 147155.

2. Aiba, T., Hesketh, G. G., Barth, A. S., Liu, T., Daya, S., Chakir, K., et al. (2009). Electrophysiological consequences 
of dyssynchronous heart failure and its restoration by resynchronization therapy. Circulation, 119, 1220-1230.

3. Arts, T., Bovendeerd, P., Delhaas, T., \& Prinzen, F. (2003). Modeling the relation between cardiac pump function and myofiber mechanics. Journal of Biomechanics, 36, 731736.

4. Arts, T., Bovendeerd, P. H. M., Prinzen, F. W., \& Reneman, R. S. (1991). Relation between left ventricular cavity pressure and volume and systolic fiber stress and strain in the wall. Biophysical Journal, 59, 93-102.

5. Arts, T., Delhaas, T., Bovendeerd, P., Verbeek, X., \& Prinzen, F. W. (2005). Adaptation to mechanical load determines shape and properties of heart and circulation: The Circ-Adapt model. American Journal of Physiology. Heart and Circulatory Physiology, 288, H1943-H1954.

6. Arts, T., Prinzen, F. W., Snoeckx, L. H., Rijcken, J. M., \& Reneman, R. S. (1994). Adaptation of cardiac structure by mechanical feedback in the environment of the cell: A model study. Biophysical Journal, 66, 953-961.

7. Arts, T., Reneman, R. S., \& Veenstra, P. C. (1979). A model of the mechanics of the left ventricle. Annals of Biomedical Engineering, 7, 299-318.

8. Bers, D. M. (2002). Cardiac excitation-contraction coupling. Nature, 415, 198-205.

9. Bovendeerd, P. H. M., Arts, T., Huyghe, J. M., van Campen, D. H., \& Reneman, R. S. (1994). Influence of endocardialepicardial crossover of muscle fibers on left ventricular wall mechanics. Journal of Biomechanics, 27, 941-951.

10. Bovendeerd, P. H. M., Arts, T., Huyghe, J. M., van Campen, D. H., \& Reneman, R. S. (1996). Regional wall mechanics in the ischemic left ventricle: Numerical modeling and dog experiments. The American Journal of Physiology, 270, H398H410.

11. Campbell, S. G., Lionetti, F. V., Campbell, K. S., \& McCulloch, A. D. (2010). Coupling of adjacent tropomyosins enhances cross-bridge-mediated cooperative activation in a Markov model of the cardiac thin filament. Biophysical Journal, 98, 2254-2264.

12. Colli-Franzone, P., Guerri, L., \& Tentoni, S. (1990). Mathematical modeling of the excitation process in myocardial tissue: Influence of fiber rotation on wavefront propagation and potential field. Mathematical Biosciences, 101, 155235.

13. Courtemanche, M., Ramirez, R. J., \& Nattel, S. (1998). Ionic mechanisms underlying human atrial action potential properties: Insights from a mathematical model. American Journal of Physiology. Heart and Circulatory Physiology, 275, H301$\mathrm{H} 321$.

14. Grandi, E., Pandit, S. V., Voigt, N., Workman, A. J., Dobrev, D., Jalife, J., et al. (2011). Human atrial action potential and $\mathrm{Ca}^{2+}$ model. Circulation Research, 109, 10551066.

15. Grandi, E., Pasqualini, F. S., \& Bers, D. M. (2010). A novel computational model of the human ventricular action potential and Ca transient. Journal of Molecular and Cellular Cardiology, 48, 112-121.

16. Greenstein, J. L., \& Winslow, R. L. (2011). Integrative systems models of cardiac excitation-contraction coupling. Circulation Research, 108, 70-84.

17. Gurev, V., Constantino, J., Rice, J. J., \& Trayanova, N. A. (2010). Distribution of electromechanical delay in the heart: Insights from a three-dimensional electromechanical model. Biophysical Journal, 99, 745-754.

18. Henriquez, C. S. (1993). Simulating the electrical behavior of cardiac tissue using the bidomain model. Critical Reviews in Biomedical Engineering, 21, 1-77.
19. Hermeling, E., Verhagen, T. M., Prinzen, F. W., \& Kuijpers, N. H. L. (2009). Transmural heterogeneity in ion channel properties in the left ventricle optimizes pump function during natural electrical activation. Computers in Cardiology, 36, $397-400$

20. Hill, A. V. (1938). The heat of shortening and the dynamic constants in muscle. Proceedings of the Royal Society of London, 126, 136-195.

21. Hodgkin, A. L., \& Huxley, A. F. (1952). A quantitative description of membrane current and its application to conduction and excitation in nerve. Journal of Physiology, 117, 500544.

22. Humphrey, J. D., \& Rajagopal, K. R. (2002). A constrained mixture model for growth and remodeling of soft tissues. Mathematical Models and Methods in Applied Sciences, 12, 407-430.

23. Huxley, A. F. (1957). Muscle structure and theories of contraction. Progress in Biophysics and Biophysical Chemistry, 7, 255-318.

24. Iyer, V., Mazhari, R., \& Winslow, R. L. (2004). A computational model of the human left-ventricular epicardial myocyte. Biophysical Journal, 87, 1507-1525.

25. Jeyaraj, D., Wilson, L. D., Zhong, J., Flask, C., Saffitz, J. E., Deschênes, I., et al. (2007). Mechanoelectric feedback as novel mechanism of cardiac electrical remodeling. Circulation, 115, 3145-3155.

26. Jie, X., Gurev, V., \& Trayanova, N. (2010). Mechanisms of mechanically induced spontaneous arrhythmias in acute regional ischemia. Circulation Research, 106, 185-192.

27. Kääb, S., Nuss, B., Chiamvimonvat, N., O'Rourke, B., Pak, P. H., Kass, D. A., et al. (1996). Ionic mechanisms of action potential prolongation in ventricular myocytes from dogs with pacing-induced heart failure. Circulation Research, 78, 262-273.

28. Keener, J. P., \& Sneyd, J. (1998). Mathematical physiology. New York: Springer.

29. Keldermann, R. H., Nash, M. P., Gelderblom, H., Wang, V. Y., \& Panfilov, A. V. (2010). Electromechanical wavebreak in a model of the human left ventricle. American Journal of Physiology. Heart and Circulatory Physiology, 299, H134-H143.

30. Keldermann, R. H., Ten Tusscher, K. H. W. J., Nash, M. P., Bradley, C. P., Hren, R., Taggart, P., et al. (2009). A computational study of mother rotor VF in the human ventricles. American Journal of Physiology. Heart and Circulatory Physiology, 296, H370-H379.

31. Kerckhoffs, R. C. P., Faris, O. P., Bovendeerd, P. H. M., Prinzen, F. W., Smits, K., McVeigh, E. R., et al. (2003). Timing of depolarization and contraction in the paced canine left ventricle: Model and experiment. Journal of Cardiovascular Electrophysiology, 14, S188-S195.

32. Kerckhoffs, R. C. P., Faris, O. P., Bovendeerd, P. H. M., Prinzen, F. W., Smits, K., McVeigh, E. R., et al. (2005). Electromechanics of paced left ventricle simulated by straightforward mathematical model: Comparison with experiments. American Journal of Physiology. Heart and Circulatory Physiology, 289, H1889-H1897.

33. Kerckhoffs, R. C. P., Healy, S. N., Usyk, T. P., \& McCulloch, A. D. (2006). Computational methods for cardiac electromechanics. Proceedings of the IEEE, 94, 769-783.

34. Kerckhoffs, R. C. P., Lumens, J., Vernooy, K., Omens, J. H., Mulligan, L. J., Delhaas, T., et al. (2008). Cardiac resynchronization: Insight from experimental and computational models. Progress in Biophysics and Molecular Biology, 97, 543561. 
35. Kerckhoffs, R. C. P., McCulloch, A. D., Omens, J. H., \& Mulligan, L. J. (2009). Effects of biventricular pacing and scar size in a computational model of the failing heart with left bundle branch block. Medical Image Analysis, 13, 362369.

36. Kerckhoffs, R. C. P., Omens, J. H., McCulloch, A. D., \& Mulligan, L. J. (2010). Ventricular dilation and electrical dyssynchrony synergistically increase regional mechanical nonuniformity but not mechanical dyssynchrony: A computational model. Circulation: Heart Failure, 3, 528-536.

37. Kohl, P., \& Sachs, F. (2001). Mechanoelectric feedback in cardiac cells. Philosophical Transactions of the Royal Society of London, 359, 1173-1185.

38. Kooshkabadi, M., Whalen, P., Yoo, D., \& Langberg, J. (2009). Stretch-activated receptors mediate cardiac memory. $P A C E, 32,330-335$.

39. Kroon, W., Delhaas, T., Arts, T., \& Bovendeerd, P. H. M. (2009). Computational modeling of volumetric soft tissue growth: Application to the cardiac left ventricle. Biomechanics and Modeling in Mechanobiology, 8, 301-309.

40. Kroon, W., Delhaas, T., Bovendeerd, P. H. M., \& Arts, T. (2009). Computational analysis of the myocardial structure: Adaptation of cardiac myofiber orientations through deformation. Medical Image Analysis, 13, 346-353.

41. Kuijpers, N. H. L., Ten Eikelder, H. M. M., Bovendeerd, P. H. M., Verheule, S., Arts, T., \& Hilbers, P. A. J. (2007). Mechanoelectric feedback leads to conduction slowing and block in acutely dilated atria: A modeling study of cardiac electromechanics. American Journal of Physiology. Heart and Circulatory Physiology, 292, H2832-H2853.

42. Kuijpers, N. H. L., Ten Eikelder, H. M. M., Bovendeerd, P. H. M., Verheule, S., Arts, T., \& Hilbers, P. A. J. (2008). Mechanoelectrical feedback as a trigger mechanism for cardiac electrical remodeling: A model study. Annals of Biomedical Engineering, 36, 1816-1835.

43. Kuijpers, N. H. L., Hermeling, E., \& Prinzen, F. W. (2010). Mechano-electrical feedback during cardiac resynchronization therapy? Computers in Cardiology, 37, 833-836.

44. Kuijpers, N. H. L., Potse, M., Van Dam, P. M., Ten Eikelder, H. M. M., Verheule, S., Prinzen, F. W., et al. (2011). Mechanoelectrical coupling enhances initiation and affects perpetuation of atrial fibrillation during acute atrial dilation. Heart Rhythm, 8, 429-436.

45. LeGrice, I. J., Smaill, B. H., Chai, L. Z., Edgar, S. G., Gavin, J. B., \& Hunter, P. J. (1995). Laminar structure of the heart: Ventricular myocyte arrangement and connective tissue architecture in the dog. The American Journal of Physiology, 269, H571-H582.

46. Lin, I. E., \& Taber, L. A. (1995). A model for stress-induced growth in the developing heart. Journal of Biomechanical Engineering, 117, 343-349.

47. Lumens, J., Arts, T., Broers, B., Boomars, K. A., Van Paassen, P., Prinzen, F. W., et al. (2009). Right ventricular free wall pacing improves cardiac pump function in severe pulmonary arterial hypertension: A computer simulation analysis. American Journal of Physiology. Heart and Circulatory Physiology, 297, H2196-H2205.

48. Lumens, J., Blanchard, D. G., Arts, T., Mahmud, E., \& Delhaas, T. (2010). Left ventricular underfilling and not septal bulging dominates abnormal left ventricular filling hemodynamics in chronic thromboembolic pulmonary hypertension. American Journal of Physiology. Heart and Circulatory Physiology, 299, H1083-H1091.

49. Lumens, J., Delhaas, T., Kirn, B., \& Arts, T. (2009). Threewall segment (TriSeg) model describing mechanics and hemodynamics of ventricular interaction. Annals of Biomedical Engineering, 37, 2234-2255.

50. Luo, C. H., \& Rudy, Y. (1994). A dynamic model of the cardiac ventricular action potential. I. Simulations of ionic currents and concentration changes. Circulation Research, 74, 1071-1096.

51. Medina-Ravell, V. A., Lankipalli, R. S., Yan, G. X., Antzelevitch, C., Medina-Malpica, N. A., Medina-Malpica, O. A., et al. (2003). Effect of epicardial or biventricular pacing to prolong QT interval and increase transmural dispersion of repolarization. Circulation, 107, 740-746.

52. Nash, M. P., \& Hunter, P. J. (2000). Computational mechanics of the heart. Journal of Elasticity, 61, 131-141.

53. Nickerson, D., Smith, N., \& Hunter, P. (2005). New developments in a strongly coupled cardiac electromechanical model. Europace, 7, S118-S127.

54. Niederer, S. A., Hunter, P. J., \& Smith, N. P. (2006). A quantitative analysis of cardiac myocyte relaxation: A simulation study. Biophysical Journal, 90, 1697-1722.

55. Niederer, S. A., Plank, G., Chinchapatnam, P., Ginks, M., Lamata, P., Rhode, K. S., et al. (2011). Length-dependent tension in the failing heart and the efficacy of cardiac resynchronization therapy. Cardiovascular Research, 89, 336-343.

56. Niederer, S. A., Shetty, A. K., Plank, G., Bostock, J., Razavi, R., Smith, N. P., et al. (2011). Biophysical modeling to simulate the response to multisite left ventricular stimulation using a quadripolar pacing lead. PACE. doi:10.1111/j.15408159.2011.03243.x.

57. Niederer, S. A., \& Smith, N. P. (2009). The role of the FrankStarling law in the transduction of cellular work to whole organ pump function: A computational modeling analysis. PLoS Computational Biology, 5, e1000371.

58. Niederer, S. A., Ter Keurs, H. E., \& Smith, N. P. (2009). Modelling and measuring electromechanical coupling in the rat heart. Experimental Physiology, 94, 529-540.

59. Nordsletten, D. A., Niederer, S. A., Nash, M. P., Hunter, P. J., \& Smith, N. P. (2011). Coupling multi-physics models to cardiac mechanics. Progress in Biophysics and Molecular Biology, 104, 77-88.

60. Nygren, A., Fiset, C., Firek, L., Clark, J. W., Lindblad, D. S., \& Clark, R. B. (1998). Mathematical model of an adult human atrial cell; the role of $\mathrm{K}^{+}$currents in repolarization. Circulation Research, 82, 63-81.

61. O’Hara, T., Virág, L., Varró, A., \& Rudy, Y. (2011). Simulation of the undiseased human cardiac ventricular action potential: Model formulation and experimental validation. PLoS Computational Biology, 7, e1002061.

62. Omens, J. H., \& Fung, Y. C. (1990). Residual strain in rat left ventricle. Circulation Research, 66, 37-45.

63. O'Rourke, B., Kass, D. A., Tomaselli, G. F., Kääb, S., Tunin, R., \& Marbán, E. (1999). Mechanisms of altered excitationcontraction coupling in canine tachycardia-induced heart failure, I: experimental studies. Circulation Research, 84, 562570.

64. Piacentino III, V., Weber, C. R., Chen, X., Weisser-Thomas, J., Margulies, K. B., Bers, D. M., et al. (2003). Cellular basis of abnormal calcium transients of failing human ventricular myocytes. Circulation Research, 92, 651-658.

65. Plotnikov, A. N., Yu, H., Geller, J. C., Gainullin, R. Z., Chandra, P., Patberg, K. W., et al. (2003). Role of L-type calcium channels in pacing-induced short-term and longterm cardiac memory in canine heart. Circulation, 107, 28442849.

66. Potse, M. (2012). Mathematical modeling and simulation of ventricular activation sequence: implications for 
CRT. Journal of Cardiovascular Translational Research. doi:10.1007/s12265-011-9343-6.

67. Potse, M., Dubé, B., Richer, J., Vinet, A., \& Gulrajani, R. M. (2006). A comparison of monodomain and bidomain reaction-diffusion models for action potential propagation in the human heart. IEEE Transactions on Biomedical Engineering, 53, 2425-2435.

68. Priebe, L., \& Beuckelmann, D. J. (1998). Simulation study of cellular electric properties in heart failure. Circulation Research, 82, 1206-1223.

69. Prinzen, F. W., Augustijn, C. H., Arts, T., Allessie, M. A., \& Reneman, R. S. (1990). Redistribution of myocardial fiber strain and blood flow by asynchronous activation. The American Journal of Physiology, 259, H300-H308.

70. Prinzen, F. W., Hunter, W. C., Wyman, B. T., \& McVeigh, E. R. (1999). Mapping of regional myocardial strain and work during ventricular pacing: Experimental study using magnetic resonance imaging tagging. Journal of the American College of Cardiology, 33, 1735-1742.

71. Prinzen, F. W., Vernooy, K., De Boeck, B. W., \& Delhaas, T. (2011). Mechano-energetics of the asynchronous and resynchronized heart. Heart Failure Reviews, 16, 215-224.

72. Rademakers, L. M., De Boeck, B. W., Maessen, J. G., \& Prinzen, F. W. (2008). Development of strategies for guiding cardiac resynchronization therapy. Heart Failure Clinics, 4, 333-345.

73. Rausch, M., Dam, A., Göktepe, S., Abilez, O. J., \& Kuhl, E. (2010). Computational modeling of growth: Systemic and pulmonary hypertension in the heart. Biomechanics and Modeling in Mechanobiology, 10:799-811.

74. Rice, J. J., \& De Tombe, P. P. (2008). Approaches to modeling crossbridges and calcium-dependent activation in cardiac muscle. Progress in Biophysics and Molecular Biology, 85, 179-195.

75. Rice, J. J., Wang, F., Bers, D. M., \& De Tombe, P. P. (2008). Approximate model of cooperative activation and crossbridge cycling in cardiac muscle using ordinary differential equations. Biophysical Journal, 95, 2368-2390.

76. Rice, J. J., Winslow, R. L., \& Hunter, W. C. (1999). Comparison of putative cooperative mechanisms in cardiac muscle: Length dependence and dynamic responses. American Journal of Physiology. Heart and Circulatory Physiology, 276, H1734-H1754.

77. Rodriguez, E. K., Hoger, A., \& McCulloch, A. D. (1994). Stress-dependent finite growth in soft elastic tissues. Journal of Biomechanics, 27, 455-467.

78. Rosenbaum, M. B., Blanco, H. H., Elizari, M. V., Lazzari, J. O., \& Davidenko, J. M. (1982). Electrotonic modulation of the T wave and cardiac memory. The American Journal of Cardiology, 50, 213-222.

79. Skalak, R., Dasgupta, G., Moss, M., Otten, E., Dullumeijer, P., \& Vilmann, H. (1982). Analytical description of growth. Journal of Theoretical Biology, 94, 555-577.

80. Sosunov, E. A., Anyukhovsky, E. P., \& Rosen, M. R. (2008). Altered ventricular stretch contributes to initiation of cardiac memory. Heart Rhythm 5, 106-113.

81. Taber, L. A., \& Chabert, S. (2002). Theoretical and experimental study of growth and remodeling in the developing heart. Biomechanics and Modeling in Mechanobiology, 1, 29-43.
82. Taber, L. A., \& Perucchio, R. (2000). Modeling heart development. Journal of Elasticity, 61, 165-197.

83. Trayanova, N., Plank, G., \& Rodríguez, B. (2006). What have we learned from mathematical models of defibrillation and postshock arrhythmogenesis? application of bidomain simulations. Heart Rhythm, 3, 1232-1235.

84. Trayanova, N. A. (2011). Whole-heart modeling: Applications to cardiac electrophysiology and electromechanics. Circulation Research, 108, 113-128.

85. Trayanova, N. A., \& Rice, J. J. (2011). Cardiac electromechanical models: From cell to organ. Frontiers in Physiology, 2, 43.

86. Tung, L. (1978). A bi-domain model for describing ischemic myocardial D-C potentials. Ph.D. thesis, MIT, Cambridge, Massachusetts, USA.

87. Ten Tusscher, K. H. W. J., Hren, R., \& Panfilov, A. V. (2007). Organization of ventricular fibrillation in the human heart. Circulation Research, 100, 87-101.

88. Ten Tusscher, K. H. W. J., Noble, D., Noble, P. J., \& Panfilov, A. V. (2004). A model for human ventricular tissue. American Journal of Physiology. Heart and Circulatory Physiology, 286, H1573-H1589.

89. Ten Tusscher, K. H. W. J., \& Panfilov, A. V. (2006). Alternans and spiral breakup in a human ventricular tissue model. American Journal of Physiology. Heart and Circulatory Physiology, 291, H1088-H1100.

90. Usyk, T. P., \& McCulloch, A. D. (2003). Electromechanical model of cardiac resynchronization in the dilated failing heart with left bundle branch block. Journal of Electrocardiology, 36, 57-61.

91. Usyk, T. P., \& McCulloch, A. D. (2003). Relationship between regional shortening and asynchronous electrical activation in a three-dimensional model of ventricular electromechanics. Journal of Cardiovascular Electrophysiology, 14, S196-S202.

92. Van Geldorp, I. E., Vernooy, K., Delhaas, T., Prins, M. H., Crijns, H. J., Prinzen, F. W., et al. (2010). Beneficial effects of biventricular pacing in chronically right ventricular paced patients with mild cardiomyopathy. Europace, 12, 223-229.

93. Vernooy, K., Cornelussen, R. N. M., Verbeek, X. A. A. M., Vanagt, W. Y. R., van Hunnik, A., Kuiper, M., et al. (2007). Cardiac resynchronization therapy cures dyssynchronopathy in canine left bundle-branch block hearts. European Heart Journal, 28, 2148-2155.

94. Vernooy, K., Verbeek, X. A. A. M., Peschar, M., Crijns, H. J. G. M., Arts, T., Cornelussen, R. N. M., et al. (2005). Left bundle branch block induces ventricular remodelling and functional septal hypoperfusion. European Heart Journal, 26, 91-98.

95. Wecke, L., Van Deursen, C. J. M., Bergfeldt, L., \& Prinzen, F. W. (2011). Repolarization changes in patients with heart failure receiving cardiac resynchronization therapy-signs of cardiac memory. Journal of Electrocardiology, 44, 590-598.

96. Weiss, J. N., Garfinkel, A., Karagueuzian, H. S., Chen, P. S., \& Qu, Z. (2010). Early afterdepolarizations and cardiac arrhythmias. Heart Rhythm, 7, 1891-1899.

97. Winslow, R. L., Rice, J. J., Jafri, M. S., Marbán, E., \& O'Rourke, B. (1999). Mechanisms of altered excitationcontraction coupling in canine tachycardia-induced heart failure, II: model studies. Circulation Research, 84, 571-586. 